\title{
Bangladesh Chars Tobacco Assessment Project (CTAP) 2018: a data note
}

\author{
Adnan M. S. Fakir ${ }^{1,2,3^{*}}$, Mustahsin Aziz², Mutasim Billah Mubde ${ }^{1}$, Afraim Karim ${ }^{1}$, Ashraf S. Khan ${ }^{1}$, Rifayat Raisa ${ }^{1}$, \\ Lubaba Ferdous Alim ${ }^{1}$ and Mayeesha Fahmin ${ }^{1}$
}

\begin{abstract}
Objectives: The Chars Tobacco Assessment Project 2018 is a holistic survey conducted in the chars (riverine islands) of Gaibandha in Northern Bangladesh, covering 985 households over 24 clusters. The survey was conducted with two objectives: (1) to assess levels of tobacco consumption and evaluate prevailing socio-economic, behavioral and health status of the chars population, and (2) to look at the effectiveness of advocacy campaigns to reduce tobacco consumption through behavioral nudges via randomized controlled trials (RCTs) in rural Bangladesh. The study site was purposively chosen due to its high tobacco consumption rate, and the geographical segregation of the chars aided in reducing spillovers for $\mathrm{RCT}$ design.

Data description: In addition to detailed information on tobacco (smoking and smokeless) consumption and perception, data was collected on: household composition, housing and plot ownership, consumption, risks and shocks coping, dowry, farm production, loans, savings and lending, labor income, asset holdings, migration and remittance, anthropometry, respiratory diseases, co-morbidities, reproductive history, risk and time preference. Unique to the dataset are carbon monoxide readings for accurate short term smoking measurement and FEV1 and PEF values for identification of long term lung damage. The data is representative only for the chars of Gaibandha.
\end{abstract}

Keywords: Chars Tobacco Assessment Project, CTAP, Bangladesh, South Asia, Chars, Smoking, Smokeless, Carbon monoxide, Smokerlyzer, COPD

\section{Objectives}

Bangladesh has one of the highest smoking rates in the world with an age-standardized smoking prevalence of above $34 \%$ among men [1]. Poor formulation and execution of tobacco control laws remain one of the prime reasons for such high smoking rates in the country [2]. The situation is further aggravated when the factor of smokeless tobacco (SLT) is taken into consideration. The data of CTAP, 2018, was collected to provide a holistic overview of the socio-economic, behavioral and health conditions of the households in the chars of Gaibandha of northern Bangladesh, with a special emphasis on understanding the effect of tobacco (smoking and smokeless) intake on various aspects of their daily lives.

\footnotetext{
*Correspondence: adnanfakir@gmail.com

1 BRAC University, 66 Mohakhali, Dhaka 1206, Bangladesh

Full list of author information is available at the end of the article
}

In particular, the baseline dataset was constructed with the following studies in mind:

1. Parental tobacco intake and increased risk of child malnutrition.

2. The effect of smoking on agricultural productivity.

3. Tobacco tug-of-war: anti-tobacco vis-à-vis tobacco sales promotion campaigns.

4. Child pregnancy and its effect on miscarriages and stillbirths.

5. Inter-generational transfer of dowry: can time change a "tradition"?

In addition to the aforementioned studies, the project also ran two randomized controlled trials (RCTs) to assess the effectiveness of two advocacy campaign interventions intended to reduce tobacco consumption of rural households: 
6. Repeated nudges through visual warning posters on the primary and secondary harmful effects of tobacco intake;

7. Record keeping of daily tobacco intake to counter non-rational discounting of individual tobacco consumption events.

Smoking status of the participants were assessed though carbon monoxide readings for more accurate measures vis-à-vis self-reported tobacco intake. Both interventions were 4 weeks long when the end-line survey was administered.

\section{Data description}

In addition to detailed information on tobacco (smoking and smokeless) consumption and perception, data was collected on: household composition, housing and plot ownership, consumption, risks and shocks coping, dowry, farm production, loans, savings and lending, labor income, asset holdings, migration and remittance, anthropometry, respiratory diseases, co-morbidities, reproductive history, risk and time preference. Unique to the dataset are carbon monoxide $(\mathrm{CO})$ readings, measured in parts per million ( $\mathrm{ppm}$ ) using the breath smokerlyzer tool, and forced expiratory volume in one second (FEV1) and peak expiratory flow (PEF) values taken using a digital spirometry machine for identification of longterm lung damage. Both tools have been previously used in earlier studies for reliable estimates [3, 4].

The $\mathrm{CO}$ readings were taken by asking respondents to exhale into the smokerlyzer after holding their breath for $15 \mathrm{~s}$. This allows a very precise short-term (past $12 \mathrm{~h}$ ) non-invasive measurement of the level of $\mathrm{CO}$ in the bloodstream due to smoking. The ability to have a verifiable measure of the intensity of smoking allows us to overcome the recall-bias inherent in reported measures of daily smoking habits [5]. The FEV1 and PEF values, on the other hand, were taken by asking the respondent to take a deep breath and exhale into the digital spirometry as hard as they could. Respondents were asked to repeat this thrice and the best values were recorded. The values can be referenced against normalized curves by height, weight and age to assess airway obstruction. While there is a growing argument in the available literature advising the use of FEV1 over PEF in measuring bronchial obstruction [6], we leave it to the researcher to decide which one to use for assessment.

\section{Sampling}

The sample for the data collection was selected based on a two-stage clustered sampling approach. In the first stage, 24 chars (clusters) from Gaibandha district were randomly selected. The list of 24 chars are provided in the questionnaire available in the Harvard Dataversesee "Data files" section below. At the second stage, from each of the chars, 42 households were chosen using a skipping factor of 3 households. After accounting for non-response rates, this sampling approach resulted in a final dataset of 985 households at the baseline. It should be noted that the questionnaire was administered in the local language, Bengali. To ensure minimal discrepancy in translation, the questionnaire was translated and reverse-translated for linguistic consistency checks during pre-testing, which was conducted in a char outside of the selected sample.

The sample size and cluster size selected for the study was based on calculations done using the optimal design software. Secondary data collected was been used as a reference for approximating the standardized effect size (equal to 0.318 ) and in order to discern an effect of $10 \%$, our sampling design provide a power of $83.5 \%$ estimated with an intra cluster correlation (ICC) of 0.07 , which was obtained from the Global Adult Tobacco Survey (GATS) dataset. End-line data and do files (for replication purposes) are available upon request after publication of the RCT studies.

\section{Further studies}

The enumerated data was then entered using CSPro and basic consistency checks executed using Stata 14. No additional processing was done to the publicly available dataset and prospective researchers using the dataset to explore particular hypothesis are expected to clean the data and run consistency checks that are specific to the study. As per the set of studies we have conducted using the dataset listed above, under "Objectives" section, our outputs indicate: (1) parental tobacco intake to negatively affect child stunting and underweight measures; (2) smoking to negatively affect agricultural productivity; (3) tobacco sales promotion campaigns to more strongly affect tobacco uptake than anti-tobacco campaigns induce cessation; (4) child marriage to lead to increased probability of miscarriages and stillbirths, and (5) dowry transfers to still be a strong tradition in the chars of Gaibandha. Finally, RCT results indicate record keeping of daily tobacco intake to be a significant advocacy strategy to induce tobacco cessation in the short run but impacts of visual nudges remain insignificant. We only succinctly mention the aforementioned findings to aware future researchers of the studies that have already been conducted using this dataset, such that it is easier for them to identify and focus on other gaps in the available literature. The findings are also, of course, open for replication using the data. We believe the dataset has potential for 
testing many other hypotheses and invite researchers to use the dataset to explore as per their interest.

\section{Data files}

Table 1 provides a technical overview of the dataset which is broken down into 33 separate files in the repository for easier handling.

\section{Limitations}

The major limitation of the study is that the data is representative only for the chars of Gaibandha, hence any findings derived from using this dataset does not hold external validity to a national level or any other dissimilar demography. Furthermore, while the dataset contains details of SLT consumption for females (the spouse of household heads as no household in the sample was

Table 1 Technical overview of data files

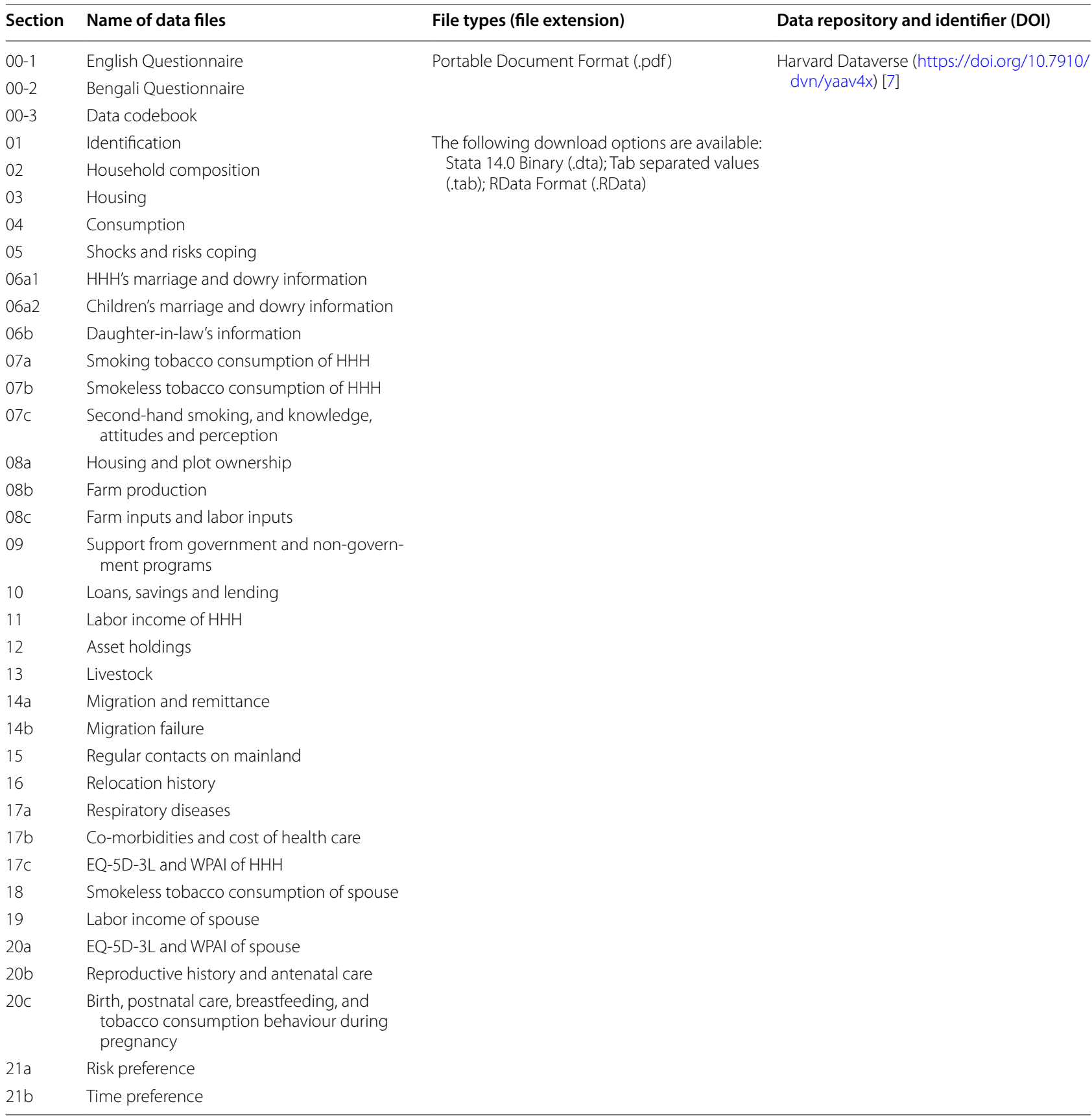


female headed), data on smoking tobacco intake was not collected for females due to cultural sensitivity. While the prevalence of such seems to be low in the sample area (based on qualitative findings not reported), this still leaves an avenue open for potential bias while conducting any comprehensive SLT analysis for females. However, if anyone wishes to investigate female vis-a-vis male SLT consumption, linking it other socio-economic factors, that remains to be explored. Finally, it should be mentioned that majority of the data are based on selfreported survey data as opposed to empirical observations (such as the CO, FEV1 and PEF values) and thus may be prone to measurement bias, as in any other field level household survey.

\section{Abbreviations}

CTAP: Chars Tobacco Assessment Project; RCT: randomized controlled trial; FEV1: forced expiratory volume in one second; PEF: peak expiratory flow; CO: carbon monoxide; COPD: chronic obstructive pulmonary disease; ICC: intra cluster correlation; CSPro: Census and Survey Processing System; SLT: smokeless tobacco.

\section{Authors' contributions}

AMSF is the principal investigator for the project and responsible for all associated studies. AMSF and MUA are responsible for the research design and all authors are responsible for designing and finalizing the questionnaire. ASK is responsible for translating the questionnaire to Bengali for use in the field. AK and MBM are responsible for designing the final CAPI questionnaire in CSPro. AMSF, MUA, ASK, MBM, AK, LFA and MF are responsible for providing enumerator training. MBM, AK, ASK, RR, LFA and MF operated as field supervisors in the data collection. RR, LFA and MF are responsible for conducting call back quality checks of the data. AMSF, AK, MBM, RR, ASK, LFA and MF are responsible for coordinating data entry, running basic consistency checks and data cleaning. All authors read and approved the final manuscript.

\section{Author details}

1 BRAC University, 66 Mohakhali, Dhaka 1206, Bangladesh. ${ }^{2}$ The World Bank, Plot E 32, Sher-e-Bangla Nagar, Agargaon, Dhaka 1207, Bangladesh. ${ }^{3}$ University of Western Australia, 35 Stirling Highway, Crawley, WA 6009, Australia.

\section{Acknowledgements}

The authors would like to firstly thank Dr. Abu S. Shonchoy for initial conversations and ideas pertaining to designing the interventions and the research proposal. The team would also like to extend gratitude to Prof. ATM Nurul Amin, Dr. Md. Kapil Ahmed and Miraz Mahmud for their support and valuable comments; MOMODa Foundation for providing the enumerators for the data collection and the students of the Economics and Social Sciences department at BRAC University for their help with the data entry process. Finally, the authors would like to acknowledge Dr. Wasiqur Rahman Khan, Dr. Rafa Faaria Alam, Naveen Abedin, Sifat Islam Ishty, Ashraf Ahmed, Maliha Rahanaz, Mowmita Haque and Nafisa Ismail for their valuable inputs during questionnaire development.

\section{Competing interests}

The authors declare that they have no competing interests.

\section{Availability of data materials}

The data described in this Data note can be freely and openly accessed on Harvard Dataverse with the following https://doi.org/10.7910/dvn/yaav4x. Please see Table 1 and reference list for details to the data.

\section{Consent for publication}

As part of the informed written consent form, the participants were specifically informed regarding open publication of the dataset for research purposes only, where any direct personal identifiers will be removed. The complete consent form can be found in the provided questionnaire of the dataset.

\section{Ethics approval and consent to participate}

Ethical approval for the survey was obtained from the review board of the James P. Grant School of Public Health at BRAC University, Ref No. 2018-028-ER.

In order to protect the right of the respondents, prior to approaching them for the survey, their written informed consent to participate in the interviews were obtained. As the demography in question contained illiterate individuals, finger stamps were obtained in some cases where the participants could not sign the written consent form. Participants were provided with full and correct information regarding the purpose of the study, nature of information required, benefits of the study and freedom to be exercised by the respondents during the enumeration. The complete consent form can be found in the provided questionnaire of the dataset.

\section{Funding}

The project was funded by Bangladesh Center for Communication Programs (BCCP) (TobaccoControl/2018-02) as part of the Johns Hopkins Bloomberg Initiative, and by BRAC University. The authors acknowledge the support of the funding bodies in the data collection process, data entry, running consistency checks of the compiled dataset and feedback in the manuscript writing process.

\section{Publisher's Note}

Springer Nature remains neutral with regard to jurisdictional claims in published maps and institutional affiliations.

Received: 4 November 2018 Accepted: 12 December 2018

Published online: 20 December 2018

\section{References}

1. Ng M, Fleming T, Robinson M, Thomson B, Graetz N, Margono C, Mullany EC, Biryukov S, Abbafati C, Abera SF, Abraham JP. Global, regional, and national prevalence of overweight and obesity in children and adults during 1980-2013: a systematic analysis for the Global Burden of Disease Study 2013. Lancet. 2014;384(9945):766-81.

2. Nargis N, Thompson ME, Fong GT, Driezen P, Hussain AG, Ruthbah UH, Quah AC, Abdullah AS. Prevalence and patterns of tobacco use in Bangladesh from 2009 to 2012: evidence from international tobacco control (ITC) study. PLoS ONE. 2015;10(11):e0141135.

3. Deveci SE, Deveci F, Açik Y, Ozan AT. The measurement of exhaled carbon monoxide in healthy smokers and non-smokers. Respir Med. 2004;98(6):551-6.

4. Erb P, Raiff BR, Meredith SE, Dallery J. The accuracy of a lower-cost breath carbon monoxide meter in distinguishing smokers from non-smokers. J Smoking Cessation. 2015;10(1):59-64.

5. Muraven M. Practicing self-control lowers the risk of smoking lapse. Psychol Addict Behav. 2010;24(3):446.

6. Nolan D, White P. FEV1 and PEF in COPD management. Thorax. 1999;54(5):468.

7. Fakir A. Bangladesh Chars Tobacco Assessment Project (CTAP). Harvard Dataverse. 2018. https://doi.org/10.7910/dvn/yaav4x (Dataset) 